\title{
A model for classical wavefront sensors and snapshot incoherent wavefront sensing
}

\author{
Congli Wang, Qiang Fu, Xiong Dun, and Wolfgang Heidrich \\ Visual Computing Center, King Abdullah University of Science and Technology, Thuwal 23955, Saudi Arabia. \\ \{congli.wang, qiang.fu, xiong.dun, wolfgang.heidrich\}@kaust.edu.sa
}

\begin{abstract}
A new formula is derived to connect between slopes wavefront sensors (e.g. Shack-Hartmann) and curvature sensors (based on Transport-of-Intensity Equation). Experimental results demonstrate snapshot simultaneous phase and intensity recovery on an incoherent illumination microscopy. () 2019 The Author(s)
\end{abstract}

OCIS codes: $010.7350,180.6900,110.1758$.

Wavefront sensors are instruments that retrieve phase $\phi(\mathbf{r})$ from wavefront-encoded intensity measurements. For incoherent light, direct wavefront sensing is not possible, and current wavefront senors are grouped into two categories: (i) slopes sensors (e.g. Shack-Hartmann [1] or Hartmann masks, lateral shearing interferometers [2], coded wavefront sensors [3-5] etc.); (ii) curvature sensors [6]. The principles of each type of sensor are usually considered separately. Here, a new theoretical model is proposed to unify slopes and curvature sensors.

A basic modeling of wavefront sensors consists of an encoding optics placed distance $z$ away from an intensity sensor, and a numerical algorithm that decodes the intensity images $\left(I_{0}(\mathbf{r})\right.$ and $\left.I(\mathbf{r})\right)$ to retrieve wavefront $\phi(\mathbf{r})$, as shown in Fig. 1(a). Different optics and customized numerical algorithms are employed for different types of

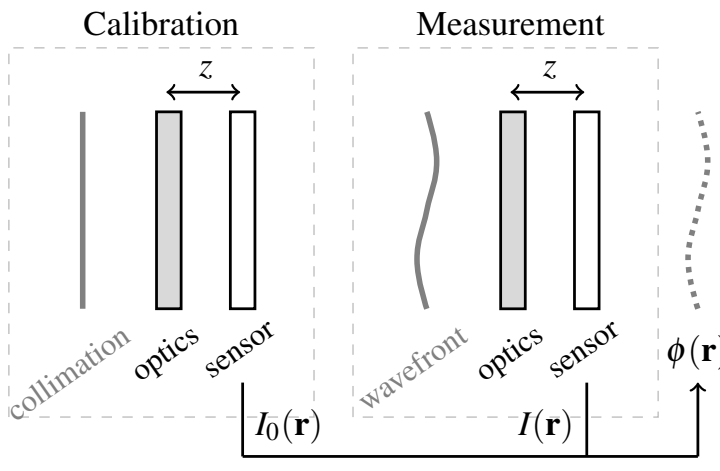

(a) General modeling of wavefront sensors.
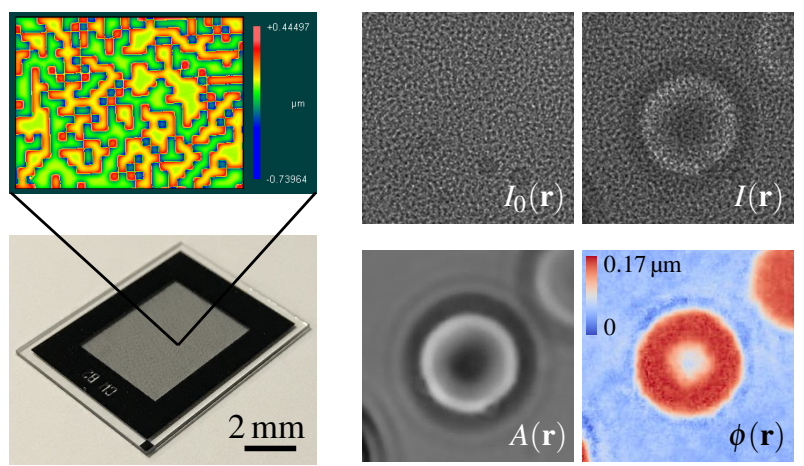

(b) The mask, typical raw images, and the numerical recovery.

Fig. 1: (a) The general model considered in this work. (b) The "optics" for our wavefront sensor (variant of [5]), and its capability to recover amplitude $A(\mathbf{r})$ and phase $\phi(\mathbf{r})$ from reference $I_{0}(\mathbf{r})$ and measurement $I(\mathbf{r})$.

wavefront sensors. Specifically in this work our wavefront sensor employs a random binary phase mask (either 0 or $\pi$ phase modulation at wavelength $550 \mathrm{~nm}$ ) as the "optics", placed $z=1.43 \mathrm{~mm}$ away from the sensor, as in Fig. 1(b). For a wavefront sensor with configuration in Fig. 1(a), at wavelength $\lambda$ (though it also works for incoherent light), one can prove, in either ray or wave optics, that the relationship between $I_{0}(\mathbf{r})$ and $I(\mathbf{r})$ is:

$$
I\left(\mathbf{r}+\frac{\lambda z}{2 \pi} \nabla \phi\right)=|A(\mathbf{r})|^{2}\left(1-\frac{\lambda z}{2 \pi} \nabla^{2} \phi\right) I_{0}(\mathbf{r})
$$

Different simplifications of Eq. (1) are the long-known principles behind various wavefront sensors as summarized in Table 1. One important case is the Transport of Intensity Equation (TIE), the principle for curvature sensors. It can be derived from Eq. (1) if linearizing $I\left(\mathbf{r}+\frac{\lambda z}{2 \pi} \nabla \phi\right)$ around $\mathbf{r}$, and two images $I_{1}(\mathbf{r})=|A(\mathbf{r})|^{2}$ and $I_{2}(\mathbf{r})=I(\mathbf{r})$ are taken at optics plane and sensor plane, respectively, and $I_{1}(\mathbf{r}) \approx I_{2}(\mathbf{r})=I(\mathbf{r})$. Movement $z \ll 2 \pi /\left(\lambda \nabla^{2} \phi\right)$ is small that $z \rightarrow 0$, justifying the partial derivative finite difference approximation.

Equation (1) is more powerful than TIE in a number of ways: (i) It is a concise theoretical model for two distanced planes, which can be separated far away (e.g. $z=1 \mathrm{~mm}$ ), whereas TIE is only valid at one particular transversal plane, and curvature sensors suffer from the finite approximation for $z$-axis derivative. As such, distance control has to be precise for example at $\mu \mathrm{m}$ scale. So it indicates our sensor outperforms curvature ones in terms of 
Table 1: Equation (1) under different forms as commonly seen formulas for each wavefront sensor. $\delta_{p}(x)$ is the Dirac comb function with period $p$ (the lenslet pitch). $k=2 \pi / \lambda$ is the wave number. Coordinate $\mathbf{r}=(x, y)$.

\begin{tabular}{|c|c|c|}
\hline Name & Optics & Model \\
\hline Shack-Hartmann & $\begin{array}{c}\text { micro-lens arrays } \\
I_{0}(\mathbf{r})=\delta_{p}(x) \delta_{p}(y)\end{array}$ & $I\left(\mathbf{r}+\frac{\lambda z}{2 \pi} \nabla \phi\right)=I_{0}(\mathbf{r})$ \\
\hline Lateral shearing & $\begin{array}{l}\text { sinusoid gratings (freq. } \omega) \\
I_{0}(\mathbf{r})=\cos ^{2}(\omega x) \cos ^{2}(\omega y)\end{array}$ & $I(\mathbf{r})=|A(\mathbf{r})|^{2} I_{0}\left(\mathbf{r}-\frac{\lambda z}{2 \pi} \nabla \phi\right)$ \\
\hline Curvature sensor & $\begin{array}{c}\text { none } \\
I_{0}(\mathbf{r})=1\end{array}$ & $\nabla I_{2} \cdot \nabla \phi+I_{1} \nabla^{2} \phi=\frac{k}{z}\left(I_{1}-I_{2}\right) \approx-k \frac{\partial I}{\partial z}$ \\
\hline Coded wavefront sensor & $\begin{array}{l}\text { random gratings } \\
I_{0}(\mathbf{r}) \text { are speckles }\end{array}$ & $I\left(\mathbf{r}+\frac{\lambda z}{2 \pi} \nabla \phi\right)=I_{0}(\mathbf{r})$ in [5]; or Eq. (1) (this work) \\
\hline
\end{tabular}

setup easiness and tolerance to $z$ inaccuracy. (ii) It contains an image warping operation (nonlinearity) and can be linearized to match the linear formulations in TIE. (iii) Our model extends the degrees of freedom for classical TIE systems to allow for a customized modulation mask (reflected as a customizable $I_{0}(\mathbf{r})$, for which in TIE is usually uniform), thus making it possible for snapshot measurements. (iv) Our model allows for broadband illumination because it is formulated in terms of optical path differences, while TIE in principle requires coherent light.

The theory reveals a potential to retrieve $|A(\mathbf{r})|^{2}$ and $\phi(\mathbf{r})$ directly from raw data. As a demonstration, by taking off the condenser, we turn an ordinary low-budget bright field microscopy into a simultaneous intensity and phase microscopy, under collimated halogen lamp (HPLS245, Thorlabs) illumination. A prototype coded wavefront sensor is employed which consists of a bare sensor (1501M-USB-TE, Thorlabs) and a mask (pixel size 12.9 $\mu \mathrm{m}$, Fig. 1(b)). Figure 2 shows simultaneous amplitude and phase recovery of transparent thin cells. Our numerical solver typically elapses within $100 \mathrm{~ms}$ on a Nvidia Titan X (Pascal) GPU for megapixel input images.

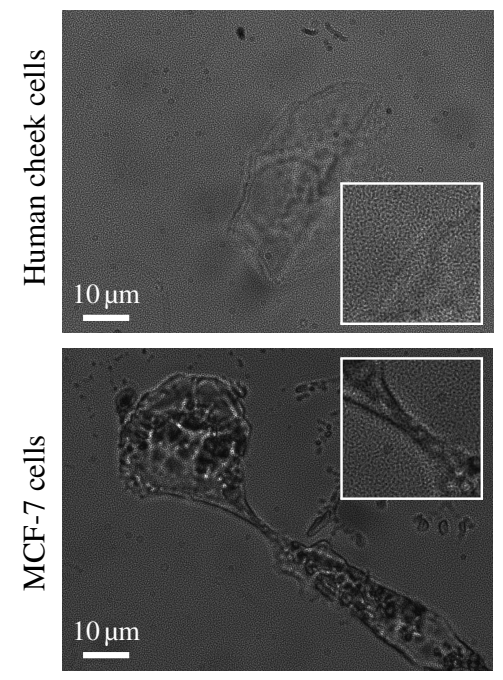

Raw data $I(\mathbf{r})$
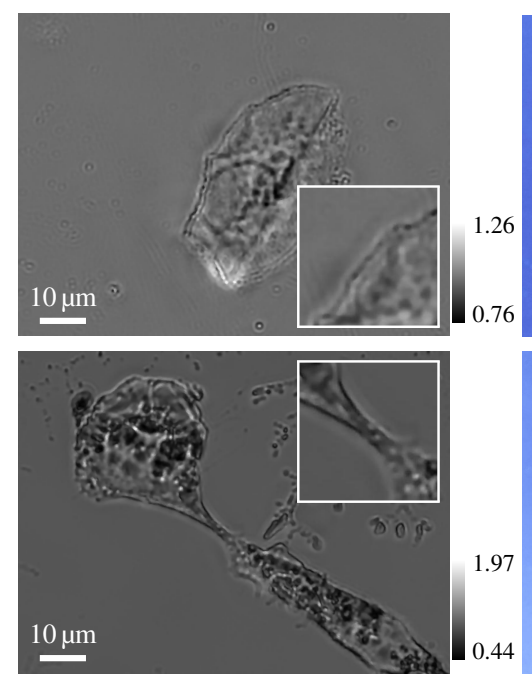

Recovered amplitude $A(\mathbf{r})$
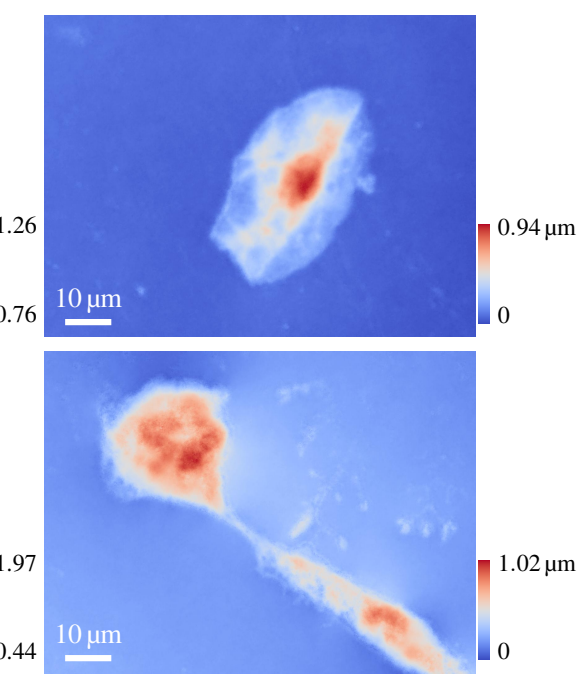

Recovered phase $\phi(\mathbf{r})$ (in OPD)

Fig. 2: Experimental results using the proposed quantitative phase imaging pipeline. Images were taken under a $\times 100$ Mitutoyo plan apochromat objective, 0.70 NA. Inset close-up images indicate that the speckle patterns have been fully removed from the original raw data. Phases are shown in terms of optical path difference (OPD).

\section{References}

1. R. V. Shack and B. C. Platt, "Production and use of a lenticular Hartmann screen," J. Opt. Soc. Am. A 61, 656 (1971).

2. P. Bon, G. Maucort, B. Wattellier, and S. Monneret, "Quadriwave lateral shearing interferometry for quantitative phase microscopy of living cells," Opt. Express 17, 13080-13094 (2009).

3. K. S. Morgan, D. M. Paganin, and K. K. Siu, "X-ray phase imaging with a paper analyzer," Appl. Phys. Lett. 100, $124102(2012)$

4. S. Bérujon, E. Ziegler, R. Cerbino, and L. Peverini, “Two-dimensional x-ray beam phase sensing," Phys. Rev. Lett. 108, 158102 (2012).

5. C. Wang, X. Dun, Q. Fu, and W. Heidrich, "Ultra-high resolution coded wavefront sensor," Opt. Express 25, 1373613746 (2017).

6. F. Roddier, "Curvature sensing and compensation: a new concept in adaptive optics," Appl. Opt. 27, 1223-1225 (1988). 